\title{
The Importance of Task Duration and Related Measures in Assessing the Distraction Potential of In-Vehicle Tasks
}

\author{
Peter Burns, Joanne Harbluk \\ Transport Canada \\ 275 Slater \\ Ottawa, On, K2H 7C8
}

peter.burns@tc.gc.ca

\author{
James P. Foley \\ Toyota Technical Center \\ 1555 Woodridge \\ Ann Arbor, Ml 48105
}

james.foley@tema.toyota.com

\author{
Linda Angell \\ Touchstone Evaluations, Inc. \\ 440 Burroughs, Box 25 \\ Detroit, Ml 48202
}

\begin{abstract}
The issue of task duration in the assessment of driver distraction has been a controversial topic. In the development of J2364 Navigation and Route Guidance Function Accessibility While Driving, task duration and a related criterion were the most difficult parts of achieving consensus. The current discussion is restricted to a few key criticisms of task duration and durationrelated measures of driving performance. We provide data-driven reasons why criticisms of duration-related measures, though important, are not sufficient to negate the value of these measures. Further, we point to naturalistic driving research that indicates it is glances away from the road scene prior to critical events that predominate in real-world crashes and near-misses. Rather than suggesting duration-related measures be abandoned, naturalistic driving research underscores the importance of using driver metrics like total eyes-off-road time as well as single glance durations. Finally, task length is an attribute of a task and HMI design, which can be modified through re-design and therefore will influence duration-related performance. We argue that duration is particularly important as a tool to assess where interventions to limit distraction might be applied.
\end{abstract}

\section{Categories and Subject Descriptors}

H.5.2 User Interfaces, Theory and Methods

\section{General Terms}

Measurement, Performance, Design, Experimentation, Human Factors, Standardization.

\section{Keywords}

Driver distraction, secondary task duration, eyes-off-road time, resumability.

The opinions and conclusions expressed or implied in this paper are those of the authors and are not necessarily those of the organization with which they are affiliated.

Copyright held by author(s)

AutomotiveUI'10, November 11-12, 2010, Pittsburgh, Pennsylvania ACM 978-1-4503-0437-5

\section{INTRODUCTION}

In the search for the most effective driving performance metrics for assessing distraction, several issues have been raised about "task duration" and duration-related measures.

Among these key issues are the following:

- Fluctuating task demand and duration-related measures;

- Duration-related measures and the variable properties of a task;

- Conceptual and practical issues with the use of task duration: task definition, task duration in dual task situations, criteria and the impact of task design on distraction; and

- Lack of evidence for resumability-based metrics.

This paper reaffirms the value of task duration and durationrelated measures for visual-manual tasks. Duration-related measures are those that co-vary to some extent with task length. Particular emphasis is given to total eyes-off-road-time or established surrogates such as Total Shutter Open Time (TSOT), as specified in ISO 16673 "Occlusion Method to Assess Visual Demand Due to the Use of In-Vehicle Systems" [1]. Task design has implications for vehicle safety, so it is important that both designers and policy makers have an accurate understanding of the issues and consistent, effective approaches to identifying and addressing the risks. Concerns about task duration are found among the recommendations from the European eSafety-HMI Working Group [2]. An open discussion of the importance of task duration is needed to acknowledge and resolve differences so that progress can be made in the development and application of useful driver metrics.

Driver metrics is a complex topic. In order to contain this discussion, it is important to establish a scope. Tasks can vary on a number of dimensions including complexity, input/output modality and duration. Visual-manual tasks require drivers to take their eyes off the road for a period of time, often repeatedly, over the course of task completion. How often and how long the eyes must be off the road and on the task is partly (though not exclusively) a function of task length. The longer the eyes are off the forward road can affect many aspects of driving safely-e.g., successful lane keeping, successful speed control and headway maintenance, as well as successful detection and response to events on the road and maintenance/updating of situation 
awareness. Therefore, given that task duration, and the aspects of task performance that vary with its duration, is particularly important for visual-manual tasks, the scope of the discussion in this paper will focus on driver-vehicle interfaces that display information visually and require manual inputs. Task duration for more cognitive, auditory or speech-based tasks is also relevant for distraction but will not be discussed herein.

In the following sections, we address the key issues given above and demonstrate that task duration and duration-related metrics are an essential consideration in the design and evaluation of invehicle information and communication systems.

\section{FLUCTUATING TASK DEMAND AND DURATION-RELATED MEASURES}

Empirical questions have been raised relating to task duration effects including both uniformity of demand over duration and cumulative effects. Fluctuating demand is an interesting challenge for driver metrics, particularly for measures based on discrete event occurrences that may or may not probe appropriate peaks and valleys of fluctuating task demand (e.g., object and event detection metrics in which any single probe may only capture a momentary slice of task demand). Improper applications of such measures (e.g., use of single probes, rather than summaries of probe responses across task periods and across participants) may not provide an indication of what occurs over the entire interaction [3] . Reliance on such measures in isolation from other measures (such as glance times and task durations) can also provide an incomplete picture. On closer examination, however, this issue of varying demand within a task does not provide sufficient basis on which to dismiss the importance and usefulness of task duration.

Task demand can fluctuate over the duration of a task. Unless a visual-manual task is very short, a driver will typically encounter several different input screens. These screens vary on their information content and choices (e.g., previous destination or new destination, keyboard screen, list of street names). The greater the quantity of information and number of choices, or input options, the more demanding it typically is for the driver to perform the task. We know from the Hick-Hyman law that response time increases with the number of available choices [4]. Complex tasks, i.e., ones that display a lot of information and options, will take more time to complete than simple tasks that have limited information and options. Thus the fluctuating demands of a task would primarily be manifested as changes in duration. It is improbable that simple and complex visual manual tasks would require the same amount of time to perform. Of course, this would depend on how complexity is defined (e.g., number of inputs, degrees-of-freedom of the control, number of choices, information content and format).

Focused visual attention is essentially a binary function. When drivers are engaged in multitasking with visual-manual tasks, they are either paying attention to the road traffic environment or fixating elsewhere (e.g., to select a menu item on a display). There may be some exceptions (e.g., the detection of emerging situations with peripheral vision), but the secondary task activities primarily involve the serial processing of visual information. If a task requires foveal attention, by definition it must distract the driver from the primary task [5]. The measurement issue is to determine the length of the distraction and consequences of the distraction on driving.

Even if a task is not uniform in its demand, a cumulative workload measure like Total Eyes Off Road Time that spans the task performance period, provides a useful summary measure of the task's overall demand. Given that a 'surprise' event can occur at any point in the task, this summary measure of load is relevant. Spikes in visual-manual task workload are likely to be manifested in longer single glances away from the road scene (up to a limit of about $2 \mathrm{~s}$ ). This highlights the importance of having both a criterion to limit long individual glances as well as a criterion to limit the total time a task takes the driver's eyes away from the road scene.

Some researchers have noted that there is some additional variance in driver responsiveness to roadway events during the task period that remains unaccounted for by total eyes off road, glance durations, and task duration. Thus they have explored augmenting the metrics toolbox with detection response metrics (also duration-related), summarized across a task period (cf. [6] and [7]). Used together, a set of such duration-related metrics can provide a comprehensive picture of task demand that is robust to and reflective of fluctuations over the period of performance.

The 100-Car Study analyses of crashes and near-miss events [8,9] indicate that the principal type of task interference that should be minimized is eye glances away from the road or Total Eyes Off Road Time. Based on considerable research, it has been concluded that Total Eyes Off Road Time limits, coupled with maximum single glance limits, are essential considerations. The Alliance of Automobile Manufacturers' DF-T guidelines provide criteria for both [10].

\section{DURATION-RELATED MEASURES AND THE INVARIANT PROPERTIES OF A TASK}

The term 'duration' can refer to several important concepts which differ from each other in critical ways.

1. Static Task Time (see SAE J2364) wherein task completion time is collected while doing nothing else (i.e., no driving or other concurrent task) [11]. This is a measure of task length, or how long a task takes to perform, when the driver's full attention is devoted to its completion. For visual-manual tasks, this measure has predictive validity for dynamic task duration (described below), number of glances to task, total glance time to task, and "speed difference" (a measure of speed variability within task) [6].

2. Task Duration While Driving (sometimes called Dynamic Task Time), where dual task interference and driving context effects can impact duration. This is a measure of task length, under divided attention (attention-switching) conditions.

3. Total Glance Time (TGT) or Total Eyes-Off-Road Time while driving. This is the cumulative time the driver looks away from the road scene during task completion while driving (see SAE J2396) [12]. This measure is not a direct measure of task length - but is a measure of visual demand that is durationrelated. As a visual-manual task increases in length, total glance times to complete the task also tend to increase. Total glance time 
is not perfectly correlated with Static or Dynamic Task Duration, but is positively related to them. This measure has been related to crash risk, as discussed later in this paper.

4. Total Shutter Open Time (TSOT), an occlusionmethod-based metric that sums the total time that vision is not occluded while the participant is completing a visual-manual task. It can be thought of as representing the total time required to "look" at a task in short " $1.5 \mathrm{~s}$ glimpses" allowed by the shutter openings in order to complete it. Predictive validity of this measure is described later.

5. Finally, there is the R-metric, calculated as the ratio of TSOT to Static Task Time, a purported indicator of the ease with which a person can stop and resume a visual-manual task (see ISO 16673 for details) [1].

The probability of being surprised by an event, and hence the risk of a conflict, increases as eyes-off-road-time accumulates over an epoch of task interaction. Visual-manual in-vehicle tasks cause drivers to look away from the road ahead for varying glance durations, typically for durations between 1.0 and 1.5 seconds, and often for multiple glances at the in-vehicle display [13, 14]. The most safety-relevant duration metric is total eyes-off-road time (or a surrogate of it). Analyses of the 100-Car Study data have shown that visual distraction is a serious concern in distraction-related crashes and close calls. Dingus, et al. [15] reported that 80 percent of all crashes and 65 percent of near crashes observed were preceded by a driver glance away from the road scene just before the onset of the conflict. Ninety three percent of rear-end crashes in the study (14 out of 15) involved a glance away from the road ahead immediately prior to the onset of the conflict.

Relative risk comparisons of various in-vehicle activities, compared to periods of 'just driving', were equally telling. Dingus and Klauer [8] found that a visual-manual activity like 'manually dialing a hand-held device' was associated with a statistically significant increase in risk (an odds ratio of 2.79). Klauer, et al. [16] reported that cumulative looks away from the road ahead of 2.0 seconds or longer within a 6 -second period prior to the onset of the conflict were associated with a statistically significant odds ratio of 2.27. Note that this is a cumulative eyes-off-road time, which is not necessarily a single glance duration.

Shutko and Tijerina [17] provided a simple quantitative model to illustrate how an increase in the number of glances away from the road can lead to an increased probability of the driver being 'surprised' by an event to which he might need to respond. A Surprise, by definition, is an unexpected (to the driver at the time) object or event.

Assume that a driver believes there will be no 'Surprises' during a glance away from the road (or else the driver would not look away at that time). Surprises nonetheless sometimes occur, and they may occur more often for inexperienced drivers who have less experience in judging changing road conditions. Then assuming the probability of a 'Surprise' is uniformly distributed throughout a task's duration, a simple probability model can be constructed that has the following form. Let the probability of a Surprise be $\mathrm{P}($ Surprise $)=\mathrm{L}$. Then the probability of no Surprise is $\mathrm{P}(\mathrm{No}$ Surprise $)=1-\mathrm{L}$. For $\mathrm{K}$ glances away, the probability of at least 1 surprise during $\mathrm{K}$ glances away will be equal to 1 minus the probability of no surprises in $\mathrm{K}$ glances away. Assuming the $\mathrm{K}$ glances away from the road are independent from one another,

$$
\begin{aligned}
& \mathrm{P}(\text { at least } 1 \text { Surprise during K glances })= \\
& 1-\mathrm{P}(\text { No surprises in } \mathrm{K} \text { glances away })= \\
& 1-(1-\mathrm{L})^{\mathrm{K}}
\end{aligned}
$$

Table 1 shows the results of this simplified model for an arbitrary value of $\mathrm{L}=0.0003$. As can be seen, there is a factor of 10 growth in the probability of the driver being surprised as the number of glances away from the road increases from 1 to 10 .

$\begin{array}{cc}\begin{array}{c}\text { Table 1. Probability of a Surprise Event as a f } \\ \text { number of glances away (simple model) }\end{array} \\ \begin{array}{c}\text { Number of Glances } \\ \text { K }\end{array} & \begin{array}{r}\text { P (At least one Surp } \\ \text { During Glance }\end{array} \\ & \\ 1 & 0.0003 \\ 2 & 0.0006 \\ 3 & 0.0009 \\ 4 & 0.0012 \\ 5 & 0.0015 \\ 6 & 0.0018 \\ 7 & 0.0021 \\ 8 & 0.0024 \\ 9 & 0.0027 \\ 10 & 0.0030\end{array}$

This demonstrates the notion that as time accumulates during which the eyes are off the road from frequent and/or long glances during the epoch of task interaction, the more likely that a driver will be surprised by a conflict. The longer a visual-manual task interaction takes to complete, the higher this cumulative risk function is likely to grow.

Dynamic task duration will be affected by the driving context [18]. As driving demands increase and the driver takes account of this increase in demand, interruptions to in-vehicle tasks will be longer. That has the effect of increasing the total duration of attention-sharing that the driver must manage while driving. The driver typically sheds the in-vehicle task and does what he or she perceives the driving situation requires. Drivers may be able to self-regulate somewhat by reducing the demands of the driving task (e.g., lowering their speed and increasing headway), but this compensation has its limits, especially given that a driver's perception of risk is impaired by distraction. Indeed, total eyesoff-road time has been shown to be less influenced by driving task demands $[18,19]$ and more influenced by the characteristics of the visual-manual task [20]. For this reason cumulative glance time away from the road (total eyes off road time) is useful to quantify the cumulative demand of a task. Other measures, such as glance rate (e.g., number of glances per unit time), fail to capture this issue of cumulative demand and the associated risk, unless they are computed for a task's duration. 
Surrogate measures that are validated as predictive of total eyesoff- road-time can be beneficial due to the difficulty of directly measuring eye glance behavior. Visual occlusion is a surrogate that has proven useful in guiding design practices to help minimize distraction. It is not a perfect replication of actual driving behavior but a useful surrogate for predicting total eyes off road. Foley [21] points out that, since the intent of the occlusion technique is to measure visual demand, the inability of occlusion to exactly mimic more intermittent natural glance behavior is not a hindrance to its application. TSOT has been demonstrated to be repeatable, discriminating, and predictive of certain on-road and test track driving performance measures (related to task duration, lane keeping and speed control, number of glances to task and total-eyes-off-road time) [6, 19]. However, TSOT does not predict single glance duration, nor responsiveness to events [6].

Finally, variation in human performance is a given, as is variation in the demands of the road traffic environment. Some aspects of a task, such as how quickly a person asymptotes in learning it, will vary task-by-task and, in this sense, may be considered a task characteristic that merits evaluation or further consideration on how tests are run.

\section{CONCEPTUAL \& PRACTICAL ISSUES WITH THE USE OF TASK DURATION.}

The use of task duration in the assessment of distraction has been criticized on the basis of assumed conceptual and practical problems. Such criticism is directed at four main points: (1) the difficulty of defining a task, (2) the difficulty of predicting task duration in a dual task situation and (3) challenges of setting criteria and 4) the impact of system design on task duration. In this section, we examine each of these issues.

\subsection{The difficulty of defining a task}

The notion that it is not possible to define a task (see Design Goal II [22]) runs contrary to basic methods of task analysis in human factors and industrial engineering. Working definitions of "task" have been developed by several groups (e.g., Alliance Guidelines; ISO; JAMA Guidelines) and are currently in use [10, 23, 24]. Although there are slight variations in wording, these definitions have in common the idea that a task is a "sequence of inputs leading to a goal at which the driver will normally persist until the goal is reached". An example of a well-defined and understood task is a driver obtaining guidance to a destination by entering a street address in a navigation system. For most in-vehicle information systems (IVIS), tasks are well-defined as such systems are designed to perform specific functions and consequently have a specific number of steps and durations associated with them.

Clearly, some tasks are more well-defined than others. Some of the tasks that drivers engage in, such as "finding something to listen to", are more loosely defined and may exhibit more of a "hunting/exploring/learning" quality. This does not mean, however, that tasks of this type cannot be defined. Tasks such as these have been observed in naturalistic driving studies (e.g., Angell, Perez, \& Hankey [7]) and analyses from naturalistic data will aid in our further understanding and characterization of these tasks.
The objection has been raised that drivers may also perform a series of individual tasks while driving. Even though it is true that tasks can be concatenated, it does not follow that task duration is invalidated if they are. Single task duration sets the lower bound for a task in any dual task situation. There is nothing to prevent a driver from performing a series of tasks. To the extent that each of those individual tasks is well designed, with short eyes-off-road duration, distraction will be reduced relative to what it would otherwise have been (if longer tasks had been concatenated).

Task duration can be measured and it offers a design opportunity that is actionable. Most visual-manual tasks with longer eyes-offroad times are not acceptable as they increase the risk of a crash. Such long tasks should be redesigned so that they are shorter and less visually intensive, requiring less eyes-off-road time, or else should be addressed through another means (use of automation for a portion of the task, use of a new innovation, or even application of a lock-out, which is an accepted practice by some manufacturers.)

\subsection{The difficulty of predicting task duration in a dual task situation}

A "task", as defined above, is associated with a time for completion. This measurement is straightforward in the case of single task duration but becomes more variable under dual task performance. What is clear, nonetheless, is that visual-manual tasks can be defined by associated measures, such as total eyesoff-road time, which are objectively collected.

Task duration assessment during design development provides a reasonable estimate of the number of glances required later without having to do the glance assessment. This task time becomes even more important when we consider that a non-trivial task undertaken while driving almost always is broken into segments of eye glances where the driver must switch visual attention back and forth between the road and the interface in order to complete the task. Task durations will vary, not only for different groups of individuals (e.g., novice and elderly drivers), but also for the same individual under different conditions. Total Glance Time away from the road is less influenced by driving conditions and more dependent upon task design, as recently demonstrated by Jahn, Krems, and Gelau [18]. We acknowledge that these systems will be used by many different people in many different scenarios, making the design imperative for short eyesoff-road times or durations all the more important.

\subsection{Criterion Setting}

An empirical basis for setting a criterion for task duration has been provided in the Alliance of Automobile Manufacturers' Driver Focus-Telematics (DF-T) Statement of Principles. Voluntary guidelines have set criterion after thorough study and vigorous debate [10]. Alliance members are following this guideline for in-scope system functions. The Guidelines contain a Total Eyes-Off-Road Time (i.e., Total Glance Time Away) limit of 20 seconds in addition to a requirement that individual glances typically not exceed $2.0 \mathrm{~s}$. The empirical bases for these limits are included in the guideline document. They are based on published literature available at the time and a reference task of radio tuning as a societally accepted level of task demand and risk. The 
Guidelines offer some of the first quantitative criteria to limit the visual distraction potential for in-vehicle interfaces.

Another example of empirically-based criterion is the JAMA Guidelines' criterion [24] for total eyes-off-road time. According to these guidelines, the operation of a display is prohibited if the task requires a total glance time in excess of 8 seconds. Using the Occlusion method, the total shutter open time shall not exceed 7.5 seconds.

\subsection{The impact of system design on task duration}

Unlike Eckstein \& Gijessl [25], we believe that good drivervehicle interface design can reduce task duration and can therefore reduce duration-related performance interference, and consequently can reduce or limit distraction and collision risk. Studies have found significant differences in performance to achieve identical goals (e.g., destination on navigation system) on different devices with different interfaces [20, 26, 27]. Task duration, as measured by TSOT, has been shown to vary dramatically and significantly as a function of driver-vehicle interface design [26]. System designers have an critical role since their decisions on system operation influence task durations. Well-designed tasks enable a driver to reach that goal more quickly and efficiently than a poorly designed task. Devices should not require or encourage the endless repetition of secondary tasks.

\section{RESUMABILITY (INTERRUPTIBILITY)}

Designing "interruptability" and "resumability" into a task is associated with the psychological concept of 'chunkability.' From a theoretical point of view, this concept implies that a interruptible task allows performance to be accomplished in small "chunks" (perhaps, but not necessarily, subtasks). These "chunks" might help prevent human short-term memory limits from being exceeded, therefore providing natural points for task-switching and attentional shifts. This might permit interruption or suspension of activity between task chunks so that attention could be devoted to the roadway, without any deleterious effects on secondary task performance when the task is subsequently resumed. Resumability implies a driver could use a strategy of task performance that involves a great deal of interleaving or switching between the primary and secondary task over time- and offers the possibility that such a strategy is hypothesized to allow the driver to focus adequately on the road in between "chunks" of the secondary task - in order to maintain sufficient situation awareness, vehicle control, and responsiveness to events. The questions about this construct include whether "interruptibility" is an attribute of task design - or whether it is a natural function of driver behavior (an epiphenomenon of natural behavior to manage both primary and secondary tasks), and, in either case, whether it, by itself, assures that a task is not distracting. These are important questions.

Regardless of the answers to these questions, one thing is clear: if a task is interrupted and resumed during its performance, its task duration will increase along with any related performance metrics (number of glances, for instance). Whether or not there are deleterious effects on driving safety may depend largely on the length of intervals between periods of task activity (the epochs during which attention is returned to the road), which may vary in length - but will determine the quality of a driver's situation awareness, responsiveness to events on the road, as well as lane keeping and speed/headway maintenance. Little attention has been paid to these concerns in research, and few of these fundamental research questions have been examined to date.

Rather, most recent work on interruptability has instead focused on whether simple surrogate measures exist to assess the construct of interruptability/resumability. Occlusion is one method that has been examined for this purpose. There are several limitations with the notion of resumability in the Occlusion Test. Resumability, and the tendency to suspend, take a break/ pause, or 'bookmark' progress mid-task may be contraindicated based on memory loads, and human motivation if it does not account for the additional burden of reorientation to the task. Miller, Galanter, and Pribram [28] pointed out that beyond forgetting where you were and what the next step is, the overall "plan" must be maintained in working memory during the pause. Moray [29] has pointed out that "...forgetting is a potent source of uncertainty and may become the dominant determinant of attention." These issues all suggest that at this juncture, research is very much needed on these fundamental questions about interruption and resumption in the context of multitasking during driving.

The definition of a task includes that "a person will persist until the goal is reached", a motivation issue which has important safety implications for task designers. Cnossen, Meijman and Rothengatter [30] reported that drivers were highly motivated to get route information while driving to the detriment of their driving performance in high demand driving situations. Resumability implies that a driver may string a task out over some long period of time which is unrepresentative of typically motivated human behaviour. Given the human propensity to complete tasks (Zeigarnik effect [31]), all tasks should be designed to allow completion in as short a time as possible and without the need for long glances. Weiner [32] has summarized the motivation results by stating that the tendency to resume an interrupted task increases if: the task has a definite goal state or purpose; the task is close to completion; the duration of interruption is short; and the person is intrinsically motivated, rather than induced, to perform a task. That said, even though we do not agree with the concept of resumability, it is still good design to tolerate interruptions in the performance of a task.. However, while it may facilitate usability -it is unknown to what degree (if any) it reduces distraction.

In this regard, it is should be noted that Dingus and Klauer [8] have recently reported that an interruptible task is still risky if it takes the eyes off the road. They state:

"...the results presented in this paper show no indication of a constant, or nearly constant, crash/near crash risk, for a broad range of in-vehicle tasks given that multiple glances away from the roadway are required. It is clear that a common crash/near crash situation involves an unexpected external event occurring when the driver is not looking in the direction of the event. It would then follow that the crash/near crash risk is greatly influenced by the joint probability of where the driver is looking and the probability of an unexpected event. Therefore, secondary tasks that require the driver to take their eyes off of the road for long and/or multiple periods will have the elevated crash/near crash risk, even if they are more easily managed by the driver." 
The selection of measures for visual-manual task demand, and corresponding criteria, is a very difficult undertaking. Any single measure will be subject to limitations and possible misinterpretation. As was highlighted in the extensive research of the CAMP Driver Workload Metrics project [33] and the EU project HASTE there [20] is no single "silver bullet." To understand workload and distraction, a variety of measures are necessary to provide complete assessment. That being said, there is also great pressure to provide simple yet effective metrics to designers for use in product development to avoid the designs that increase, rather than minimize driver distraction.

The appropriate application of occlusion and other metrics should continue to be studied. Occlusion can be used to calculate a measure of resumability known as the R-metric. $\mathrm{R}$ is the ratio of TSOT and Static Task Time (measured under un-occluded conditions) -- a purported indicator of the ease with which a person can stop and resume a visual-manual task (see ISO 16673 for details) [1]. This is based on the notion that the numerator reflects the consequences of visual interruption from shutter closures of the occlusion device. However, another way to think about this ratio is that this calculation (with "looking time (TSOT) in the numerator, and task duration in the denominator) represents that percentage of task duration that was spent "looking" at a task during its performance (using the shutter-open glimpses) something quite different from resumability. Though based on occlusion metrics, the R-metric is thus somewhat analogous to "Percent Total Eyes Off Road" in the road-based domain - and yet, the R-metric does not correlate significantly with eyes off road time in CAMP DWM research [6] and related research.

Occlusion can be used to assess secondary tasks, but by itself it is not sufficient [34]. In the CAMP research TSOT had a 0.92 correlation with mean total duration of all glances (TGT). It was concluded that Median TSOT predicts the following measures (with $\mathrm{R}^{2} \sim 0.50$ or more): Task time when driving; Standard Deviation of Lane Position (SDLP); Speed Difference between task start and end; TGT and Counts of Task-Related (TR) Glances away from the road scene. Hashimoto and Atsumi [35] in a research paper to support the JAMA guidelines report a correlation of .893 between TSOT and TGT (as measured during on-the-road driving). Thus TSOT provides a robust measure that can be used for laboratory assessments.

However, the usefulness of the resumability measure $\mathrm{R}$ is not supported by the data and is less accepted by experts in this field than TSOT. More research support is needed before it can be used as an effective design tool. This issue is raised by Foley [21] and supported by CAMP DWM research report by Shutko and Tijerina [17] where it was found that $\mathrm{R}$ does not correlate well with any eye glance measures. The $\mathrm{R}$ metric is unrelated to OnRoad and Test-Track driving performance measures in the CAMP research. Burns, in summarizing several different occlusion studies, stated that it is "unclear what R measures" and that mean $R$ values were almost always less than 1 . Only one task on the 8 real systems tested had a mean R greater than 1 (1.09) and the lowest $R$ value was 0.59 [36].

There is no perfect single measure for identifying distraction. Naturalistic studies of crash risk have shown total eyes-off-road time to be a good predictor of crash risk. However, it is a difficult measure to collect and cannot easily be used during the early stages of the product cycle to assist in making good decisions for the driver vehicle interface design. Research is needed to establish more surrogate measures for total eyes-off-road time and/or to utilize other duration-related measures in combination with it.

\section{CONCLUSION}

Any metric that ignores task duration and duration-related metrics in the assessment of visual-manual tasks will have an incomplete, and possibly misleading, estimation of distraction risk. As concluded by the CAMP DWM [33] and HASTE [20] projects, any given single measure of distraction provides an incomplete assessment of distraction. However measures that are empirically supported, such as task duration, and total eyes off road time, can and should be used to aid driver vehicle interface designers in making good decisions as early as possible in the design process. Once the interface has reached the prototype stage, changes in design are more difficult and expensive, therefore less likely to be made. To completely assess the potential for driver distraction several measures are required for a reliable driver metric and task duration should be reflected in at least one of them. Research is needed to establish more surrogate measures for total eyes-offroad.

Research is also needed to further examine the impact that task design and duration has on safe driving performance. Naturalistic driving research is a powerful approach for capturing large quantities of real world data on driver behavior [14, 15]. Many of these research questions may be answered in the near future. The second Strategic Highway Research Program (SHRP 2) will conduct a naturalistic driving study of unprecedented scale [37]. Sensors will be installed on the vehicles of 4,000 volunteer drivers over 2 years in multiple sites across the United States. Naturalistic data from additional sites will be collected in a Canadian project as well. These data will provide insights into the safety implications of duration for visual-manual tasks as well as speech-based tasks. Data from SHRP 2 will help to augment our understanding of driver behavior based on empirical evidence.

\section{REFERENCES}

[1] International Standards Organization, ISO 16673 Road Vehicle - Ergonomic Aspects of Transport Information and Control Systems-Occlusion Method to Assess Visual Demand Due to the Use of In-Vehicle Systems, Geneva, Switzerland, 2007.

[2] eSafety-HMI Working Group, (2005). Recommendations from the eSafety-HMI Working Group: Final Report, European Commission, Information Society Technologies, Paris 28, 2005.

[3] Victor, T.W., Engström, J., \& Harbluk, J.L. (2009). Distraction Assessment Methods Based on Visual Behavior and Event Detection. In Driver Distraction: Theory, Effects and Mitigation. (M.A. Regan, J.D. Lee, \& K.L. Young Eds.) pp. 135-165

[4] Hyman, R. Stimulus Information as a Determinant of Reaction Time. Journal of Experimental Psychology, 1953, 45, 188-196.

[5] Wierwille, W. W. (1993). Demands on Driver Resources Associated with Introducing Advanced Technology into the Vehicle, Transportation Research, 1 (2), 133-142. 
[6] Angell, L. S. (2007). Effects of Seconary Task Demands on Drivers' Resonses to Events during Driving: surrogate Methods \& Issues. 4th International Driving symposium on Human factors in Driver assessment, Training and Vehicle Design: Driving Assessment2007 Conference. Stephenson, Washington.

[7] Angell, L.S., Perez, M., \& Hankey, J. (2008). Driver Usage Paterns for Secondary Information Systems. The First Human Factors symposium on Naturalistic Driving Methods \& Analyses, August 25-28, 2008, Virginia Tech Transportation Institute, Blacksburg, Virginia.

[8] Dingus. T., and Klauer, S. (2008). The relative risks of secondary task induced driver distraction (Paper No. 200821-0001). Warrendale, PA: Society of Automotive Engineers. Paper presented at Convergence 2008, Detroit, MI. October 20-22, 2008.

[9] Klauer, S.G. Guo, F., Sudweeks, J. and Dingus, T.A. (May 2010) An Analysis of Driver Inattention Using a CaseCrossover Approach on 100-Car Data: Final Report DOT HS 811334.

[10] Statement of Principles, Criteria, and Verification Procedures on Driver Interactions with Advanced In-Vehicle Information and Communications Systems, Draft Version 2.1 (with updates), Driver Focus-Telematics Working Group, Alliance of Automobile Manufacturers, June 2006.

[11] SAE Recommended Practice for Navigation and Route Guidance Function Accessibility While Driving, SAE J2364.

[12] SAE Definitions and Experimental Measures Related to the Specification of Driver Visual Behavior Using Video Based Techniques, SAE J2396.

[13] Victor, T. (2005) Keeping Eye and Mind on the Road. PhD thesis. Uppsala Universitet.

[14] Hanowski, R.J., Perez, M.A., and Dingus, T.A., Driver distraction in long-haul truck drivers, Transportation Research Part F: Traffic Psychology and Behaviour 8(6), 441-458, 2005.

[15] Dingus, T. A., Klauer, S.G., Neale, V. L., Petersen, A., Lee, S. E., Sudweeks, J., Perez, M. A., Hankey, J., Ramsey, D., Gupta, S., Bucher, C., Doerzaph, Z. R., Jermeland, J., and Knipling, R.R. (2006). The 100-Car Naturalistic Driving Study, Phase II - Results of the 100-Car Field Experiment (DOT HS 810 593). Washington, DC: U.S. Department of Transportation, National Highway Traffic Safety Administration.

[16] Klauer, S., Dingus, T., Neale, V., Sudweeks, J. and Ramsey, D. (2006). The Impact of Driver Inattention on Near/Crash Risk: An Analysis Using the 100-Car Naturalistic Driving Study Data. (DOT HS 810 594). Washington, DC: U.S. Department of Transportation, National Highway Traffic Safety Administration (NHTSA).

[17] Shutko, J., and Tijerina, L. (2006). Eye glance behavior and lane exceedences during driver distraction. Presentation given at Driver Metrics Workshop, Ottawa, October, 2006. Web site http://ppc.uiowa.edu/drivermetricsworkshop/.

[18] Jahn, G., Krems, J. F., and Gelau, C. (2009). Skill Acquisition While Operating In-Vehicle Information
Systems: Interface Design Determines the Level of SafetyRelevant Distractions. Human Factors, Vol. 51, No. 2, April 2009, pp. 136-151.

[19] Tijerina, L., and Kochhar, D. (2007). A Measurement Systems Analysis of Total Shutter Open Time (TSOT) as a Visual-Manual Task Distraction Metric. Proceedings of the Human Factors and Ergonomics Society 51st Annual Meeting, Baltimore, MD, October 3, 2007. (CD)

[20] Carsten, O., Merat, N., Janssen, W., Johansson, E., Fowkes, M., and Brookhuis, K., HASTE Final Report, Contract No. GRD1/2000/25361 S12.319626, Human Machine Interface and the Safety of Traffic in Europe (HASTE) Project, 2005.

[21] Foley, J. Now You See It Now You Don't: Visual Occlusion as a Surrogate Distraction Measurement Technique in Regan, M., Lee, J. and Young, K. (Eds.) Driver Distraction Theory, Effects and Mitigation, CRC Press, Boca Raton, 2009.

[22] ESOP (2008). Commission Recommendation of 26 May 2008 on safe and efficient in-vehicle information and communication systems: update of the European Statement of Principles on human-machine interface, Official Journal of the European Union, 2008/653/EC.

[23] Road vehicles - Ergonomic aspects of transportation information and control systems - Simulated lane change test to assess in-vehicle secondary task demand, ISO/DIS 26022, November 2008.

[24] Japan Automobile Manufacturers Association Guideline for In-vehicle Display Systems (JAMA), 2004. www.jamaenglish.jp/release/release/2005/Invehicle_Display_GuidelineVer3.pdf

[25] Eckstein, L. and van Gijssel, A. (2006). HMI Guidelines and the Effect on Process, Product and Traffic Safety (Rep. No. SAE 2006-01-0574). SAE International.

[26] Harbluk, J. L., Burns, P. C., Go, E., \& Morton, A. H. (2006) The occlusion procedure for assessing in-vehicle telematics: Tests of current vehicle systems. In Proceedings of the Human Factors and Ergonomics Society 50th Annual Meeting (pp. 2373-2377). Santa Monica, CA: Human Factors and Ergonomics Society.

[27] Harbluk, J.L., Mitroi, J.S, \& Burns, P.C. (2009). Three navigation systems with three tasks: Using the lane change test (LCT) to assess distraction demand. Proceedings of the 5th International Driving Symposium on Human Factors in Driver Assessment, Training, and Vehicle Design. Big Sky, Montana, June 2009.

[28] Miller, G., Galanter, E., and Pribram, K. (1966/1986). Plans and the structure of behavior. Adams Bannister Cox Pub.

[29] Moray, N. (1993). Designing for attention. In A. Baddeley and L. Weiskrantz (Eds.), Attention: Selection, awareness, and control (pp. 111-134). Oxford: Clarendon Press.

[30] Cnossen F, Meijman T, Rothengatter T. (2004). Adaptive strategy changes as a function of task demands: a study of car drivers. Ergonomics. 47(2), 218-36.

[31] Zeigarnik, B.V. (1967). On finished and unfinished tasks. In W. D. Ellis (Ed.), A sourcebook of Gestalt psychology, New York: Humanities Press. 
[32] Weiner, B. (1972). Theories of motivation: From mechanism to cognition (pp. 143-144). Chicago: Markham.

[33] L. Angell, J. Auflick, P.A. Austria, D. Kochhar, L. Tijerina, W. Biever, T. Diptiman, J. Hogsett, and S. Kiger, CAMP Driver Metrics Workload Project Task 2 Final Report, DOT HS 810 635, Washington, DC 2006

[34] Noy, Y.I., Lemoine, T.L., Klachan, C., and Burns, P.C., Task interruptibility and duration as measures of visual distraction, Applied Ergonomics, 35, 2004, 207.

[35] Hashimoto, K. and Atsumi, B. 2001, Study of occlusion technique for making the static evaluation method of visual distraction, Exploring the occlusion technique: progress in recent research and applications workshop Torino, Italy,
Sept 2001,

(http://www.umich.edu/ driving/occlusionworkshop2001).

[36] Burns, P. C. R Occlusion Research. Presented at the Driver Metrics Workshop, Ottawa, June 2006.

[37] TRB Special Report 296, Implementing the Results of the Second Strategic Highway Research Program (SHRP 2): Saving Lives, Reducing Congestion, Improving Quality of Life, Transportation Research Board, Washington, D.C. 2009.Ding, W. and Marchionini, G. 1997. A Study on Video Browsing Strategies. Technical Report. University of Maryland at College Park. 